\title{
High-resolution satellite image segmentation using Hölder exponents
}

\author{
Debasish Chakraborty ${ }^{1, *}$, Gautam Kumar $\mathrm{Sen}^{2, * *}$ and Sugata Hazra ${ }^{2, \dagger}$ \\ ${ }^{1}$ Regional Remote Sensing Service Centre, Indian Space Research Organization, IIT Campus, \\ Kharagpur 721 302, India. \\ ${ }^{2}$ School of Oceanographic Studies, Jadavpur University, Kolkata 700 032, India. \\ *e-mail:deba.isro@gmail.com deba1964@yahoo.com \\ **e-mail: gksju@yahoo.co.in \\ †e-mail: shazra@geology.jdvu.ac.in
}

\begin{abstract}
Texture in high-resolution satellite images requires substantial amendment in the conventional segmentation algorithms. A measure is proposed to compute the Hölder exponent (HE) to assess the roughness or smoothness around each pixel of the image. The localized singularity information is incorporated in computing the HE. An optimum window size is evaluated so that HE reacts to localized singularity. A two-step iterative procedure for clustering the transformed HE image is adapted to identify the range of HE, densely occupied in the kernel and to partition Hölder exponents into a cluster that matches with the range. Hölder exponent values (noise or not associated with the other cluster) are clubbed to a nearest possible cluster using the local maximum likelihood analysis.
\end{abstract}

\section{Introduction}

The spectral or pixel-based segmentation technique consists of K-Means (Hartigan et al 1979), Fuzzy C Means (Bezdek et al 1984) and methods of Minimum Distance (Richards 1995), which considers only the spectral pattern to segment the image. These techniques are not sufficient to segment high-resolution satellite images due to the variability of spectral and structural information in such images. Thus the spatial pattern or texture analysis becomes necessary to segment highresolution images.

\subsection{Relevant studies on high-resolution image segmentation}

High-resolution image segmentation technique deals with watershed approach, region growing, and simulated annealing.
The watershed segmentation methods (Vincent and Soille 1991; Debeir 2001; Wang et al 2004) transform the original data into a gradient image. The local 'minima' of the gradient image, which are pre-selected seeds, are regarded as 'catchment basins'. All points that 'flow' into the same 'catchment basins' are divided into the same watershed. In the process of segmenting, the regions surrounding the seeds become 'flooded' and many regions grow out to a point where they can contact with each other. Finally, the image is classified into separate 'catchments' bordered by the watershed lines. In this approach, the number of 'catchments' is equal to the number of these seeds. Wang et al (2005) had further improved this method by incorporating the texture information of the image together with the gradient information to segment the image. The main drawback of the method is, choice of more seed points leads oversegmentation while too few seeds possibly lead to under-segmentation.

Keywords. High resolution image; texture analysis; segmentation; IKONOS; Hölder exponent; cluster. 
Region-growing procedure (Bins et al 1996; Carleer et al 2005) starts at each seed point in the image with one-pixel objects, and smaller image objects are merged into bigger ones, throughout a pair-wise clustering process based on three criteria: color, smoothness, and compactness. The balance at which these criteria are applied depends on the desired output. If the smallest growth exceeds a heterogeneity tolerance defined by the user, the process stops. The heterogeneity tolerance affects the relative size of output polygons. The main shortcoming of the method is that it over-segments the homogeneous areas and mostly under-segments the textured areas such as forests.

Simulated annealing (Cook et al 1996) is an iterative procedure, starts with an initially segmented image and randomly changes the current state. The annealing then decides whether or not to accept the new configuration by calculating the difference between the likelihood of the data fitting these segmentations. The method is used to segment the image of small structures, but it often leads to faulty segmentation. It often does oversegmentation to represent the boundaries of low contrast area as well as boundaries of sufficient contrast area (e.g., forest vs. meadow).

Some more techniques like fractal analysis (Kaplan 1999; Voorons et al 2003), Minimum Entropy approaches (Hermes and Buhmann 2001), etc., have been used for segmenting high spatial resolution remote sensing images. But the proper way of segmenting all type of features from such high spatial resolution images are required to be developed, because the use of these methods on images leads either to under-segmentation or to over-segmentation (Meinel and Neubert 2004).

\subsection{Hölder exponent}

Texture reflects the spatial structure of pixel values and hence plays an important role in segmenting high-resolution satellite images. But due to very high local variability of the pixels value in those images, the characterization of textures becomes difficult. Various methods like cooccurrence matrices (Haralick et al 1973; Tsai et al 2005), Markovian tools (Chellappa and Chatterjee 1985), gabor filters (Turner 1986; Guo et al 2000) are used to describe textures, but suffer due to their limitations to identify many texture features. Recently LBP is used to identify the texture in the image. LBP (Local Binary Patterns) is a technique that describes the texture in terms of both statistical and structural characteristics. Gray scale rotation invariant (Ojala et al 2000) is a technique used for texture analysis based on LBP and nonparametric discrimination of sample and prototype distributions. But it is not suitable to discriminate textures where the dominant features exist on a large scale. Therefore, the Multivariate Texture analysis (Lucieer et al 2005) combines the rotation invariant variance (VAR) with LBP to analyze the texture. But the performance of this technique is not satisfactory since it considers only a part of the surrounding of pixels for measuring texture of the image.

In last two decades the Hölder exponent (HE) has been used for texture analysis of images including synthetic aperture radar images (Bourissou et al 1994; Levy-Vehel and Mignot 1994; Liu and Li 1997; Malladi et al 2003; Tahiri et al 2005). The central concept of Hölder exponent analysis is that it measures the local regularity of the image. Advantages of using $\mathrm{HE}$ on high-resolution images are that

- it can be used as a tool to measure the roughness or smoothness around each pixel in the image, and

- it does not require any prior information about the pixel intensity. This work attempts to describe the textures of high-resolution images using Hölder exponent.

Definition of Hölder exponent (Bourissou et al 1994): Let $\mu$ be a measure on a set $\Omega$ as well as for all $x \in \Omega, \ni \alpha(x)$, such that $\mu\left(B_{r}(x)\right) \sim r^{\alpha}$, for small $r$. Here $B_{r}(x)$ is a circle (2D) of radius $r$ centered on $x$. Then $\alpha(x)$ is called the Hölder exponent on $x$.

In case of an image, the Hölder exponent $\alpha(x, y)$ is a function of the pixel $(x, y)$. Naturally it could be assumed that by calculating each pixel's Hölder exponent based on a pre-defined measure, new representation of an original image could be obtained. For a 2D image denoted by $(x, y, I(x, y))$ where $I(x, y)$ represents the intensity of the pixel $(x, y)$, its Hölder exponent representation could be expressed as $(x, y, \alpha(x, y))$ where $\alpha(x, y)$ represents the Hölder exponent value of the pixel $(x, y)$.

Since the Hölder exponent is used to transform the image, the transformed image is called Hölder exponent image. This Hölder exponent image is a combination of set of textures. Further, the similar type of textures together form the texturesegmented image.

\subsection{Outline of the proposed work}

A new measure is proposed to compute the Hölder exponent to asses the roughness or smoothness around each pixel in the image. The localized singularity is considered to include only the singularity information during the computation of HE. An optimum window size is evolved to 
get localized singularity. To show the robustness of the Hölder exponent analysis the K-mean is applied separately on the transformed images obtained from GLCM analysis as well as images obtained from the Hölder exponent analysis and compared the results. From the results, it is found that the 'HE analysis and K-mean' gives less heterogeneous segments than 'GLCM and K-mean'. But it could not segment all classes distinctly. Yet another technique is the adaptive method, which uses reinforcement learning to learn the reward values of successive data clustering. It applies only when external feedback exists for a clustering task (Bagherjeiran et al 2005). Therefore a clustering technique is proposed to segment the HE image. The proposed method depends on the mean-shift (Comaniciu and Meer 2002; Di et al 2003; Chakraborty et al 2008) technique. It is used to identify the range of HE densely occupied in the kernel and partition those Hölder exponent into a cluster that matches with the range. To show the significance of the contribution, the proposed clustering method is compared with the K-mean. Moreover, the experimental results show that the use of K-mean method can superpose vegetation, fallow, built-up area and bare soil, consequently it decreases the classification accuracy of the image while the proposed clustering technique mostly overcomes these discrepancies. The proposed method is proved to be effective to segment IKONOS 1m PAN images.

The paper is organized as follows. In section 2, a detailed methodology including description of new measure, localized singularities, proposed image transformation and description of new segmentation method are discussed. Comparison of the result of

- Gray Level Co-occurrence Matrix (GLCM) analysis and K-mean,

- Hölder exponent analysis and K-mean, and

- Hölder exponent analysis and proposed clustering

is discussed in section 3. The final conclusions are drawn in section 4.

\section{Methodology}

Methodology adapted to segment high-resolution images has two main steps:

- image transformation, and

- clustering.

In the first step each pixel of the original image is transformed into a measure of texture on the basis of its neighbour, while the transformed image is clustered in the second step.

\subsection{Image transformation}

The Hölder exponent analysis is used here to transform the image for the identification of the texture. It does not require any prior information about the pixel intensity. The predefined measure is used to estimate the degree of texture around each pixel.

\subsubsection{Measures}

The pre-defined measure is one of the most important characteristics to compute the Hölder exponent (Bourissou et al 1994; Liu and Li 1994). The roughness or smoothness around each pixel can be assessed by the appropriate estimation of the measure. In this paper we determine the measure of dispersion of pixel values using linear regression analysis.

Let the subset $\Omega^{*}$ of the region $\Omega$ contains only those pixels which intersect the perimeter of the circle of radius $r$. Hence for $t$ number of increasing radius (i.e., $r=1$ to $t$ ) there will be $t$ number of subsets $\Omega^{*}$. Subsequently the radius $r$ versus the intensity values $I(i)$ of that subset $\Omega^{*}$ is plotted and from the least square fit of regression line calculate the intensity value $J$ for each radius $r$. As a result, a new measure $K(i)=|I(i)-J|$, for each $i \in \Omega^{*}$ is obtained. In turn this provides the dispersion of pixels from the line of regression. The above measure can be represented as:

$$
\begin{aligned}
& \mu_{\mathrm{disp}}\left(\Omega^{*}\right)=\{K(i)=|I(i)-J| ; \operatorname{Min}(I(i)) \\
&\leq J \leq \operatorname{Max}(I(i))\}
\end{aligned}
$$

where $J$ is the derived intensity value for radius $r$ using the regression equation.

$\mu_{\text {disp }}\left(\Omega^{*}\right)$ is the measure of dispersion of pixels contained in the subset $\Omega^{*}$.

\subsubsection{Hölder exponent analysis}

In this subsection, the Hölder exponent $\alpha(x, y)$ of each pixel $(x, y)$ over the whole image is calculated. As described in the definition, for each pixel, a series of measure of balls centered on this pixel with incremental radius values are obtained. Logarithmic plots of computed measure $(K)$ versus radius $(R)$ values are drawn and got the Hölder exponent $\alpha$ as follows:

$$
\alpha=\frac{1}{N} \sum_{r=1}^{t} \sum_{i=1}^{m_{r}} \frac{\log K(i)}{\log R(r)},
$$


where $t$ is the total number of identified balls, $m_{r}$ is the number of intersected pixel on the perimeter of the circle of radius $R(r)$ and $N$ is the total number of pixels under each ball of radius $R(r)$.

In this study for each pixel a series of 15 values of radius (i.e., $1, \sqrt{ } 2, \sqrt{ } 5,3, \sqrt{ } 13,3 \sqrt{ } 2,5, \sqrt{ } 29,2 \sqrt{ } 10$, $3 \sqrt{ } 5,7, \sqrt{ } 61,6 \sqrt{ } 2, \sqrt{ } 85,7 \sqrt{ } 2)$ are taken. Values of radius whose differences are less than the threshold value are considered as the same radius. This is done to minimize the computation complexity. The maximum value of radius up to $7 \sqrt{ } 2$ has been used, so that $\alpha$ reacts to localized singularities.

\subsubsection{Optimum window size for localized singularities}

Assessment of window size is one of the important issues to study the localized singularities. $\mathrm{Gu}$ et al (2008) have used separability measure to find the window size for extracting residential area from IKONOS $1 \mathrm{~m}$ panchromatic image. Chakraborty et al (2008) have used density of range of pixel values to find the window size for clustering the IRS 1C/1D 6 m PAN images. Yashon et al (2008) have used semivariogram fitting to find the optimal window size for the land cover classification of $70 \mathrm{~cm}$ PAN QuickBird imagery. In this study, the window size is assessed to detect the localized singularities. The larger window size is insensitive to noise that leads to loss of information of singularity, while the smaller window size represents the singularity well, but sensitive to noise. So it is preferable to determine the window from two respects:

- additional singularity should not be contained in the same window; so the window should be diminished to keep the singularity,

- the size of the window should be enlarged on the locations without obvious singularity.

Considering all the above factors, measure of dispersion of pixels from the line of regression is used here as a standard for choosing the size of window. Consequently it calculates the average dispersion of pixel values from the line of regression of ten (user defined) windows (selected randomly in the image) and determines whether the opted larger size window is small enough, i.e., the difference with the smallest average dispersion is small enough than the appointed threshold. The opted size of window is adopted for computation of localized singularities. Otherwise, it determines the smaller size of window and finds again whether the difference between the smallest average dispersion is small enough than the appointed threshold, then this size of window is adopted. This technique has been applied in multiple images and found $15 \times 15$ size of window is optimum for most of the case to obtain the localized singularities.

\subsection{Clustering of HE image}

An iterative clustering procedure is adapted to:

- detect the range $\left(R_{Q}\right)$ of a cluster contained in the kernel based on a preliminary estimated HE range,

- localize the cluster center, and

- identify the cluster contained in the kernel $G$.

\subsubsection{Detection of the range $\left(R_{Q}\right)$ of a cluster in the Hölder exponent image}

The range $R_{Q}$ of a cluster in the Hölder exponent image is defined as follows:

Let us consider $G=\left\{g_{k l}\right.$, Hölder exponent value in $G(k, l)$, where $k=1, \ldots, m$ and $l=1, \ldots, m\}$ is a kernel with $m^{2}$ Hölder exponent (HE). $Q$ is a cluster in $G$ with center $C_{Q}$ (mean). Then the range $R_{Q}$ of the cluster $Q$ contains only those HE values satisfying the following properties:

$$
\operatorname{Abs}\left(g_{k l}-C_{Q}\right)<R_{Q} .
$$

Equation 3 means that cluster $Q$ contains that range of $\mathrm{HE}$ value, which have a minimum degree of association (represented by $R_{Q}$ ).

A preliminary estimated HE range $R_{Q}$ is computed before the assessment of optimal range of the cluster. It is a three-step procedure:-

- computation of mean $(G)$,

- computation of maximum $(G)$, and

- estimation of preliminary $\mathrm{HE}$ range = abs(computation of mean $(G)$-computation of $\operatorname{maximum}(G))$.

\subsubsection{Localization of cluster center}

The objective of this subsection is to find a center in the dataset where the 'density' (or number) of range of pixel values in $G$ within a range, i.e., $R_{Q}$ is locally maximal.

Primarily the cluster center is initialized with the mean HE values. We then select the HE values within the $R_{Q}$ from the center of $G$ (i.e., mean of $G$ ). Iteratively, the mean of this range of HE values is again calculated and subsequently the kernel center is moved to this mean. This approach moves the range of $\mathrm{HE}$ values in the direction where the 'density' is higher.

This is implemented iteratively by decreasing $R_{Q}$ with a constant value until absolute difference between the initial center $\left(C_{Q}\right)$ and present center (ME) reaches the desired value (minimum difference). In the first iterations (when $R_{Q}$ is still large) this technique moves the range of $\mathrm{HE}$ values 
to regions of the data where the 'global' density is higher (these regions often contain the large number of pixels). After some iteration (when $R_{Q}$ is equal to constant value) the kernel center moves towards an actual range of $\mathrm{HE}$ values where the density is 'locally' higher. Convergence is reached if the kernel center remains stationary. If this does not happen within a certain number of iterations then last computed $\mathrm{ME}$ is considered as $C_{Q}$.

\subsubsection{Identification of the cluster contained in the kernel}

The Cluster identification consists of two parts:

- Background: These are the HE values in the HE image not included between $\left(C_{Q}-R_{Q}\right)$ and $\left(C_{Q}+R_{Q}\right)$ values. Such HE values, either belongs to another cluster or do not belong to any cluster (noise; are not significantly associated with other HE values). HE values belonging to other clusters are not considered at the time of threshold calculation for the current cluster.

- Range: These are the HE values represented as $\left(C_{Q}-R_{Q}\right) \leq \mathrm{HE} \leq\left(C_{Q}+R_{Q}\right)$. $\mathrm{HE}$ values belonging to the cluster are significantly correlated.

\subsection{Maximum likelihood analysis}

The image so obtained after adopting previously mentioned clustering technique consists of noise. The classification accuracy of the image decreases due to inclusion of those noises. A method is proposed in this section to club those noises to a cluster that is spatially nearer and likelihood of occurrence is more. The method is a two-step procedure. In the first step, it computes the weight of each cluster residing in the kernel, while the maximum weighted cluster is identified in the second step.

(i) Cluster weight is computed with the formula

$$
W t_{-} \text {Cluster }_{k}=\frac{\text { Freq }_{k}}{\left(n^{*} m\right)}
$$

where $k$ is the number of cluster resides in the kernel.

Freq ${ }_{k}$ is the total number of HE falling in the range of $k$ th cluster residing in the kernel.

$W t_{-}$Cluster $_{k}$ is the possibility (or weighting factor) to assign the HE value in the $k$ th cluster.

$n$ and $m$ represent the number of row and column of the kernel respectively.

(ii) Maximum weighted cluster is identified with the equation

$$
\begin{gathered}
\operatorname{Max}_{-} W T_{-} \text {Cluster }=\left(\sup \left\{W t_{-} \text {Cluster }_{k}\right\},\right. \\
k=1 \text { to } L),
\end{gathered}
$$

where $L$ is the number of cluster contained in the kernel.

\section{Results and discussion}

To show the robustness of the proposed HE analysis the GLCM (gray level co-occurrence matrix) is used in this study. This is done by applying the K-mean separately on the transformed images obtained from GLCM analysis as well as images obtained from the Hölder exponent analysis and comparing the results of 'GLCM and K-mean' and 'HE analysis and K-mean'. Simultaneously the results of the proposed segmentation method, i.e., 'HE analysis and proposed clustering' are also compared with those two results to show the robustness of the proposed segmentation method.

The GLCM analysis (TEX software of Geomatica 10.1) is carried out by considering the five of Haralick texture parameters: entropy, dissimilarity, contrast, homogeneity and the correlation and five filter parameters: filter $\mathrm{X}$ size 5, filter $\mathrm{Y}$ size 5, number of gray levels 32 , spatial relationship $\left(P_{x}\right) 1$ and spatial relationship $\left(L_{n}\right) 0$. The distance parameter in GLCM specifies the scale at which the texture is analyzed. A particular distance provides perfect results for a particular texture pattern but not for various texture patterns in the image. Therefore, the distance is a limit factor in GLCM to identify various texture patterns in an image. The GLCM is constructed separately for each input images (as shown in figure 1a and 1e) by considering the distance of 5,7 and 9 pixels away respectively along the vertical and horizontal direction. And it is found that at distance 5 GLCM represents better textures in these images. Therefore in this case the distance of 5 pixels is considered as optimum to find the texture in the image.

'GLCM and K-mean', 'HE analysis and K-mean' and the 'Proposed segmentation' (i.e., 'HE analysis and Proposed clustering') have been applied on two different $1 \mathrm{~m}$ PAN (IKONOS) images. First image (figure 1a) is a mixing of

- vegetation,

- built-up area, and

- fallow.

Whereas second image (figure 1e) comprises of

- bare soil,

- vegetation,

- fallow, and

- built-up area. 

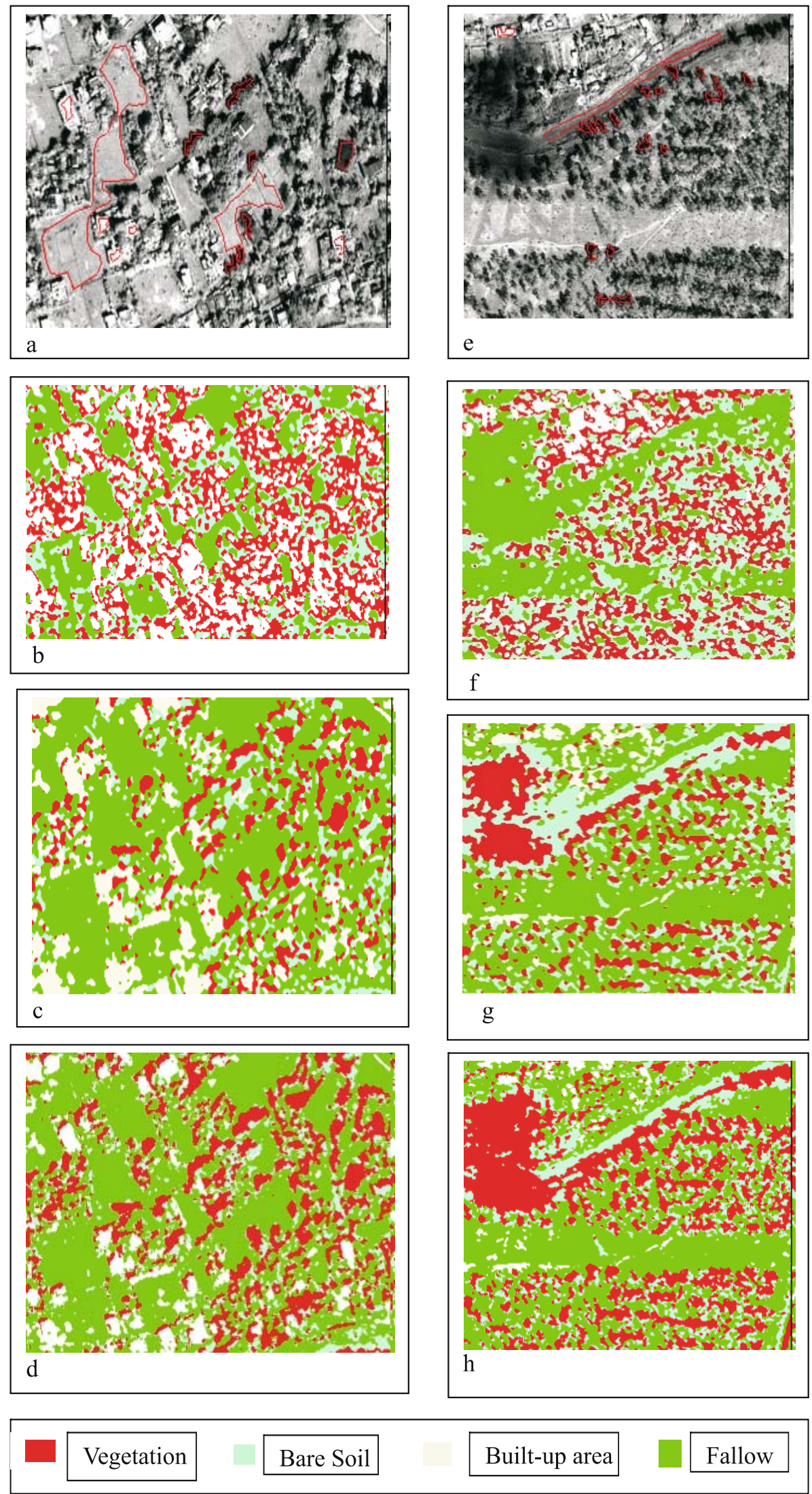

Figure 1. (a) IKONOS image of vegetation, built-up area and fallow, (b) Output image obtained by applying 'GLCM and K-mean' on (a), (c) Output image obtained by applying 'proposed transformation and K-mean' on (a), (d) Output image obtained using 'proposed transformation and clustering' on (a), (e) IKONOS image of bare soil, vegetation and fallow, (f) Output image obtained by applying 'GLCM and K-mean' on (e), (g) Output images obtained by applying 'proposed transformation and K-mean' on (e), and (h) Output image obtained using 'proposed transformation and clustering' on (e). 
Table 1. The comparative success rate for classifying the features obtained by applying (i) 'GLCM and K-mean', (ii) 'Proposed transformation (i.e., HE analysis) and K-mean' and (iii) 'Proposed transformation and proposed segmentation' separately on IKONOS images. The column of the table represents the percentage (\%) of area occupied by the features according to the segmentation results when there is a unique feature as per the ground truth.

\begin{tabular}{|c|c|c|c|c|c|}
\hline \multirow[b]{2}{*}{$\begin{array}{l}\text { Segmentation } \\
\text { method }\end{array}$} & \multirow{2}{*}{$\begin{array}{l}\% \text { Area occupied by } \\
\text { the class as per seg- } \\
\text { mentation results }\end{array}$} & \multicolumn{4}{|c|}{ Identified ground truth features } \\
\hline & & $\begin{array}{l}\text { Vegetation } \\
\quad(100 \%)\end{array}$ & $\begin{array}{l}\text { Built-up } \\
(100 \%)\end{array}$ & $\begin{array}{l}\text { Fallow } \\
(100 \%)\end{array}$ & $\begin{array}{c}\text { Bare soil } \\
(100 \%)\end{array}$ \\
\hline \multirow{4}{*}{$\begin{array}{l}\text { GLCM and K-mean } \\
\text { (figure } 1 \mathrm{~b} \text { and } 1 \mathrm{f} \text { ) }\end{array}$} & Vegetation & 23.21 & 28.21 & 5.27 & 3.30 \\
\hline & Built-up area & - & 57.71 & 4.18 & - \\
\hline & Fallow & 57.28 & 14.08 & 84.23 & 92.34 \\
\hline & Bare soil & 19.51 & - & 6.32 & 4.36 \\
\hline \multirow{4}{*}{$\begin{array}{l}\text { Proposed transfor- } \\
\text { mation and K-mean } \\
\text { (figure } 1 \mathrm{c} \text { and } 1 \mathrm{~g} \text { ) }\end{array}$} & Vegetation & 85.23 & 3.65 & 3.52 & 2.0 \\
\hline & Built-up area & - & 81.52 & 2.71 & - \\
\hline & Fallow & 9.27 & 14.83 & 91.28 & 2.85 \\
\hline & Bare soil & 5.50 & - & 2.49 & 97.15 \\
\hline \multirow{4}{*}{$\begin{array}{l}\text { Proposed transfor- } \\
\text { mation and clustering } \\
\text { (figure 1d and } 1 \mathrm{~h} \text { ) }\end{array}$} & Vegetation & 92.59 & 4.23 & 4.75 & 2.21 \\
\hline & Built-up area & - & 92.37 & - & - \\
\hline & Fallow & 4.52 & 2.32 & 93.25 & 2.50 \\
\hline & Bare soil & 2.89 & 1.08 & 2.0 & 93.29 \\
\hline
\end{tabular}

Texture is visible in both the images. The results of the proposed segmentation method (taking the kernel size of $(15,15))$ then compared with the results obtained from the analysis based on 'GLCM and K-mean', 'HE analysis and K-mean' respectively.

The outputs of the 'GLCM and K-mean', 'HE analysis and K-mean' and 'HE analysis and proposed clustering' methods after being applied on (i) first IKONOS image are shown in figure 1(b), 1(c), 1(d), (ii) second IKONOS image are shown in figure $1(\mathrm{f}), 1(\mathrm{~g})$ and $1(\mathrm{~h})$ respectively. From the results, it clearly appears that the 'HE analysis and K-mean' method gives less heterogeneous segments than 'GLCM and K-mean'. While 'HE analysis and proposed clustering' method give more homogeneous segments with distinct classes than 'HE analysis and K-mean'.

Using the ground truth data overlaid separately on the resultant outputs obtained after by means of 'GLCM and K-mean', 'HE analysis and Kmean' and 'HE analysis and proposed clustering' methods, the statistics of the classification rates for each approach is shown in table 1 . The numerical results show that the success rate for recognizing vegetation, built-up, fallow and bare soil are (23.21, $57.71,84.23$ and 4.36 ) by 'GLCM and K-mean', (85.23, 81.52, 91.28 and 97.15$)$ by the 'HE analysis and K-mean' and (92.59, 92.37, 93.25 and 93.29) by the 'proposed transformation and clustering' approach.

The experimented results with the two input images (figure 1a and 1e) through 'GLCM and K-mean' technique show under-segmentation and the observer discrepancies in this method are as follows:

- fallow and bare soil mixed with the vegetation,

- vegetation and fallow mixed with the built-up area,

- vegetation, built-up area and bare soil mixed with the fallow shown in figure 1(b), fallow and vegetation mixed with bare soil shown in figure $1(\mathrm{f})$.

This discrepancy decreases the success rate of recognizing vegetation, fallow and built-up area and bare soil as shown in table 1 whereas the 'HE analysis and K-mean' overcome these discrepancies to a certain extent. It is found that the superposition of vegetation, fallow, built-up area and bare soil becomes less as shown in figure 1(c) and 1(g). Though the decreased discrepancy increases the success rate of recognizing vegetation, fallow and built-up area in 'HE analysis and K-mean', but the 'Proposed transformation (i.e., HE analysis) and Clustering' approach mostly overcome these discrepancies (table 1). The experimental result shows that:

- vegetation is detected well and do not intermix much with fallow and bare soil,

- built-up area and fallow is classified well as shown in figure $1(\mathrm{~d})$,

- bare soil is also detected very well as shown in figure 1(h).

Consequently the success rate of recognizing vegetation, fallow and built-up area and bare soil increases more than $92 \%$ as shown in table 1 . 


\subsection{Comparison between K-mean and proposed segmentation technique}

K-mean needs number of class as an input to segment the image whereas proposed technique need not require any input for segmentation.

$\mathrm{K}$-mean only considers the information of pixel value for segmentation. The proposed segmentation technique also considers association of $\mathrm{HE}$ values to increase the classification accuracy.

In K-mean method, an iteration procedure is carried out until all pixels get classified in the image whereas in the proposed technique, iteration is done to identify the range of HE densely occupied in the kernel, and to partition those Hölder exponent into a cluster which matches with that range. Hölder exponent values (noise or not associated with the other cluster) are clubbed into a nearest possible cluster using the local maximum likelihood analysis.

Proposed segmentation method is simple and takes very less computation time while $\mathrm{K}$-mean is time consuming.

\section{Conclusion}

In this paper, the Hölder exponent is used as a tool to utilize the spatial and spectral information together to compute the degree of texture around each pixel in the high-resolution panchromatic images. The measure of dispersion $\left(\mu_{\text {disp }}\right)$ is employed to compute the Hölder exponent. A clustering procedure including maximum likelihood analysis is used to classify the Hölder exponent image. From the results of the experiments it is found that the proposed method adequately segment complex images containing texture regions as well as non-texture regions. Moreover, it can be considered as an intuitively appealing, unsupervised (no need for a predefinition of the number of clusters) and fast clustering algorithm. As a result the method is potentially useful to classify highresolution panchromatic images more efficiently and accurately.

\section{Acknowledgements}

Authors are thankful to the Director, RRSSCNNRMS for his encouragement and Indian Space Research Organization (ISRO) for providing the required satellite images. Authors are grateful to the respected unknown reviewers for their valuable advice and suggestions. Authors gratefully acknowledge the Head, RRSSC, Kharagpur for giving his support during the study.

\section{References}

Bagherjeiran A, Christoph F E and Vilalta R 2005 Adaptive Clustering: Better Representatives with Reinforcement Learning; Technical Report Number UH-CS-05-06, Department of Computer Science, University of Houston (available at http://www.cs.uh.edu/docs/preprint/uhcs-05-06.pdf).

Bezdek J C, Ehrlich R and Full W 1984 FCM: The Fuzzy c-Means clustering algorithm; Computers and Geosciences 10 191-203.

Bins L S, Fonseca L M G, Erthal G J and Ii F A M 1996 Satellite imagery segmentation: A region growing approach; Proceedings of VIII Brazilian Remote Sensing Symposium, Salvador, Bahia: $4 \mathrm{p}$.

Bourissou A, Pham K and Levy-Vehel J 1994 A Multifractal Approach for Terrain Characterization and Classification on SAR Images; IGARSS 3 1609-1611.

Chellappa R and Chatterjee S 1985 Classification of Textures using Gaussian Markov Random Fields; IEEE Transactions on Acoustics, Speech, and Signal Processing 33 959-963.

Carleer A P, Debeir O and Wolff E 2005 Assessment of Very High Spatial Resolution Satellite Image Segmentations; Photogrammetric Engineering $\&$ Remote Sensing 71(11) 1285-1294.

Cook R, McConnell I, Stewart D and Oliver C J 1996 Segmentation and simulated annealing; SPIE Microwave Sensing and Synthetic Aperture Radar 2958 30-35.

Chakraborty D, Sen G, Hazra S and Jeyaram A 2008 Clustering for high resolution monochrome satellite image segmentation; Int. J. Geoinformatics 41.

Comaniciu D and Meer P 2002 Mean Shift: A Robust Approach Toward Feature Space Analysis; IEEE Transactions on Pattern Analysis and Machine Intelligence 24(5) 603-619.

Debeir O 2001 Segmentation Supervisée d'Images; Ph.D., Faculté des Sciences Appliquées, Université Libre de Bruxelles.

Di Kaichang, Wang Jue, Ma Ruijin and Li Ron 2003 Automatic shoreline extraction from high resolution IKONOS satellite imagery; ASPRS Conference proceedings, Anchoragc, Alaska, Available at http://shoreline.eng. ohio-state.edu/publications/di_asprs03.pdf.

PCI Geomatics, 2009, Geomatica 10.1, Available at http://www.pcigeomatics.com/.

Guo G-D, Li S, Chan K and Pan H 2000 Texture Image Segmentation using Reduced Gabor Filter Set and Mean Shift Clustering; Fourth Asian Conference on Computer Vision (ACCV'00) 198-203.

Gu J, Chen J, Zhou Q M and Zhang H W 2008 Quantitative textural parameter selection for residential extraction from high-resolution remotely sensed imagery; The International Archives of the Photogrammetry, Remote Sensing and Spatial Information Sciences 37 B4.

Haralick R M, Shanmugan K and Dinstein I 1973 Textural features for image classification; IEEE Trans. on Systems, Man, and Cybernetics 6 610-621.

Hartigan J A and Wong M A 1979 A K-means clustering algorithm; Appl. Stat. 28(1) 100-108.

Hermes L and Buhmann J M 2001 A New Adaptive Algorithm for the Polygonization of Noisy Imagery; Technical Report IAI-TR-2001-3, Dept. of Computer Science III, University of Bonn.

Kaplan L M 1999 Extended Fractal Analysis for Texture Classification and Segmentation; IEEE Transaction on Image Processing 8(11) 1572-1585.

Levy-Vehel J and Mignot P 1994 Multifractal segmentation of images; Fractals 2 371-377. 
Liu Y and Li Y 1997 New approaches of multifractal image analysis; Proceedings of International Conference on information, communications and signal processing $\mathbf{2}$ 970-974.

Lucieer A, Stein A and Fisher P 2005 Multivariate texture-based segmentation of remotely sensed imagery for extraction of objects and their uncertainty; Int. J. Remote Sens. 26(14) 2917-2936.

Meinel G and Neubert M 2004 A comparison of segmentation programs for high resolution remote sensing data; International Archives of Photogrammetry and Remote Sensing 35 1097-1105.

Malladi R K, Kasilingam D and Costa A H 2003 Speckle filtering of SAR images using Hölder regularity analysis of the sparse code; IEEE Int. Geosci. Remote Sens. Symp. 6 3998-4000.

Ojala T, Pietikäinen M and Mäenpää T 2000 Gray Scale and Rotation Invariant Texture Classification with Local Binary Patterns; Proceedings of the 6th European Conference on Computer Vision 1 404-420.

Richards J A 1995 Remote Sensing Digital Image Analysis: An Introduction; Springer-Verlag 265-290.

Turner M R 1986 Texture discrimination by Gabor functions; Biological Cybernetics 55 71-82.

Tsai F, Chou M J and Wang H H 2005 Texture Analysis of High Resolution Satellite Imagery for Mapping Invasive Plants; Int. Geosci. Remote Sens. Symp. 4 3024-3027.
Tahiri A M, Farssi S M and Touzani A 2005 Textures in images classification using a multifractal approach; IEEE SITIS (Available at http://www.ubourgogne.fr/SITIS/05/download/Proceedings/Files/ f138.pdf).

Voorons M, Germain M and B'eni'e G B 2003 Segmentation of high resolution images based on the multifractal analysis; IEEE (available at http://ieeexplore.ieee.org/iel5/ 9010/28606/01294844.pdf).

Vincent L and Soille P 1991 Watershed in digital spaces: An efficient algorithm based on immersion simulations; IEEE Transactions on Pattern Analysis and Machine Intelligence 13 583-598.

Wang Z, Zhao S and Chen X 2004 Watershed segmentation of high-resolution remotely sensed data; Proc. Int. Symp. Remote Sens. 2004 \& 20th Anniversary of the Korean Society of Remote Sensing 107-109.

Wang Z, Song C, Wu Z and Chen X 2005 Improved watershed Segmentation algorithm for high resolution remote sensing images using texture; In: Proc. IEEE Int. Geosci. Remote Sens. Symp., Soul, Korea 5 3721-3723.

Yashon O O, Tetuko J and Tateishi R 2008 Analysis of cooccurrence and discrete wavelet transform textures for differentiation of forest and non-forest vegetation in veryhigh-resolution optical-sensor imagery; Int. J. Remote Sens. 29(12) 3417-3456. 